\title{
Cobertura dos Sistemas de Informação dos Cânceres do Colo do Útero e de Mama no Brasil, 2008-2019
}

doi: https://doi.org/10.32635/2176-9745.RBC.2022v68n1.1544

\author{
Cervical and Breast Cancer Brazilian Information System Coverage, 2008-2019 \\ Cobertura de los Sistemas de Información sobre Cánceres del Cuello Uterino y de Mama en Brasil, 2008-2019
}

Jeane Tomazelli'; Caroline Madalena Ribeiro'; Maria Beatriz Kneipp Dias ${ }^{3}$

RESUMO

Introdução: Ações de controle dos cânceres do colo do útero e de mama têm sido monitoradas no Brasil por meio da utilização de dados disponíveis nos sistemas de informação que registram exames de rastreamento e investigação diagnóstica desses cânceres. Objetivo: Avaliar a cobertura dos sistemas de informaçáo para o controle dos cânceres do colo do útero e de mama do Brasil. Método: Estudo transversal utilizando dados de exames citopatológicos do colo do útero, histopatológicos do colo do útero e de mama, e mamografias, registrados no Siscolo, Sismama, Siscan e SIA/SUS, segundo Região e Unidade Federativa entre 2008-2019. Calcularam-se as proporçōes anuais de exames informados apenas no SIA/SUS e realizou-se uma análise descritiva entre as Regióes e ao longo dos anos. Resultados: A cobertura foi maior para exames citopatológicos (perda de 20\% no período) e menor para histopatológico de mama (perda de 37,1\% no período). A partir de 2015, verificou-se menor cobertura para todos os exames e, em 2019, redução nos patamares de perda. Conclusáo: A cobertura dos sistemas variou no período, sendo maior para citopatológicos do colo do útero, o que indica que o tempo é um importante fator na consolidação dos sistemas.

Palavras-chave: programas de rastreamento; sistemas de informação em saúde; estudos de avaliação; Sistema Único de Saúde; indicadores (estatística).

\section{ABSTRACT}

Introduction: Cervical and breast cancer control have been monitored in Brazil through the use of data available in the information systems recording screening and diagnostic investigation tests results. Objective: To evaluate the coverage of cervical and breast cancer control information systems in Brazil. Method: Cross-sectional study using data from cytopathological cervical exams, cervix and breast histopathology exams and mammograms recorded at Siscolo, Sismama, Siscan and SIA/SUS according to region and federative unit between 2008-2019. Annual proportions of exams informed only at SIA/SUS were calculated and descriptive analysis was performed among regions and over the years. Results: The coverage was higher for cytopathological cervical exams (loss of $20 \%$ in the period) and lower for histopathological breast exams (loss of $37.1 \%$ in the period). As of 2015, there was less coverage for all exams and in 2019 there were reduction of loss levels. Conclusion: The coverage of the systems varied over the period, being higher for cytopathologic cervical exams indicating that timing is an important factor of consolidation of the systems.

Key words: mass screening; health information systems; evaluation study; Unified Health System; indicators (statistics).
RESUMEN

Introducción: Las acciones de control de los cánceres de cuello uterino y de mama han sido monitoreadas en Brasil mediante el uso de datos disponibles en los sistemas de información que registran los exámenes para detectar estos cánceres. Objetivo: Evaluar la cobertura de los sistemas de información para control del cáncer de cuello uterino y de mama en Brasil. Método: Estudio transversal que utiliza datos citopatológicos cervicales, histopatológicos cervicales y de mama y mamografías registradas en Siscolo, Sismama, Siscan y SIA/SUS según la región y Unidad Federativa entre 2008-2019. Se calcularon las proporciones anuales de los exámenes informados en el SIA/SUS y realizo análisis descriptivo entre regiones y lo largo de años. Resultados: La cobertura fue mayor para los exámenes citopatológicos ( $-20 \%$ en el período) y menor para histopatología mamaria (-37,1\% en el período). Después de 2015, hubo menos cobertura para todos los exámenes y, en 2019, una reducción en los niveles de pérdida. Conclusión: La cobertura de los sistemas varió durante el período, siendo mayor para la citopatología cervical, lo que indica que el tiempo es un factor importante en la consolidación de los sistemas.

Palabras clave: tamizaje masivo; sistemas de información en salud; estudio de evaluación; Sistema Único de Salud; indicadores (estadística).

\footnotetext{
1,2,3 Instituto Nacional de Câncer José Alencar Gomes da Silva (INCA). Rio de Janeiro (RJ), Brasil. E-mails: jtomazelli@inca.gov.br; cribeiro@inca.gov.br; mdias@inca.gov.br. Orcid iD: https://orcid.org/0000-0002-2472-3444; Orcid iD: https://orcid.org/0000-0003-2690-5791; Orcid iD: https://orcid.org/0000-0002-5847-9830

Endereço para Correspondência: Jeane Tomazelli. Rua Marquês de Pombal, 125, 70 andar - Centro. Rio de Janeiro (RJ), Brasil. CEP $22230-240$. E-mail: jtomazelli@inca.gov.br
} 


\section{INTRODUÇÃO}

No Brasil e no mundo, diversas estratégias de detecção precoce dos cânceres do colo do útero e de mama têm sido adotadas com objetivo de reduzir sua incidência e mortalidade. Apesar disso, ainda se observam elevadas taxas, principalmente nos países de baixa e média rendas. Para cada ano do triênio 2020-2022, no Brasil, estimam-se 16.590 novos casos de câncer do colo do útero e 66.280 de câncer de mama ${ }^{1}$.

Países com programas de rastreamento organizado monitoram indicadores de cobertura, qualidade dos exames e seguimento dos casos identificados como forma de avaliar e qualificar seus programas ${ }^{2-5}$. No Brasil, a partir da implantação de um programa-piloto de rastreamento do câncer do colo do útero, realizado na década de 1990, identificou-se a necessidade de obter dados para acompanhamento dos resultados do programa, tendo sido desenvolvido, em 1999, o Sistema de Informação do Controle do Câncer do Colo do Útero (Siscolo), para registro dos exames de rastreamento (exame citopatológico) e confirmação diagnóstica (exame histopatológico), realizados no Sistema Único de Saúde (SUS), além de informações sobre o seguimento das mulheres com exames alterados. Em 2009, foi desenvolvido o Sistema de Informação do Controle do Câncer de Mama (Sismama) para o registro de mamografias, exames citopatológicos e histopatológicos, além das informaçôes de seguimento ${ }^{6}$.

Em 2013, com objetivo de aperfeiçoar e integrar os sistemas, foi desenvolvido o Sistema de Informação de Câncer (Siscan), uma versáo web que permite a identificação da usuária por meio do número do cartáo SUS, facilitando assim o seguimento das mulheres com exames alterados no rastreamento, sobretudo pelas unidades de saúde informatizadas que passam a utilizar o sistema ${ }^{7,8}$. Esses sistemas também fornecem dados para a geração do Boletim de Informaçáo Ambulatorial necessário ao pagamento dos procedimentos (exames) realizados no SUS ${ }^{6,8}$.

Desde a implantação desses sistemas, seus dados têm sido utilizados para subsidiar a gestáo do programa e a análise crítica do cenário desses cânceres no sistema público do país, e para a elaboração de publicações científicas na área ${ }^{9-17}$.

Entre os problemas relatados nos estudos quanto ao uso das informaçóes dos sistemas de informação para o controle do câncer, está a perda de dados, inferida pela diferença entre o número de registros desses sistemas em relação ao de faturamento ${ }^{18-20}$, e que impacta na sua cobertura $^{21}$. Apesar de a literatura reconhecer o impacto dessas diferenças para uma adequada avaliação das ações de controle dos cânceres do colo do útero e de mama, não há estudos que mensurem a diferença de registros entre o Siscolo, o Sismama e o Siscan e o Sistema de Informaçóes Ambulatoriais do SUS (SIA/SUS) para pagamento dos procedimentos realizados.

O objetivo do presente estudo foi avaliar a cobertura do Siscolo, do Sismama e do Siscan em relação ao sistema de faturamento do SIA/SUS nas Unidades Federativas (UF) do Brasil entre 2008 e 2019.

\section{MÉTODO}

Estudo transversal utilizando dados de produção de procedimentos de rastreamento e investigação diagnóstica dos cânceres do colo do útero e de mama registrados nos seguintes sistemas: SIA/SUS, Siscan, Siscolo e Sismama. Os dados foram agrupados pela UF do prestador de serviço (laboratório ou clínica radiológica) e por ano, para o período entre 2008 e 2019. Tal período de análise foi estabelecido considerando a implementaçáo do registro individualizado de procedimentos ambulatoriais, boletim de produção ambulatorial (BPA-I), na tabela de procedimentos do SUS ${ }^{22}$, utilizados nos procedimentos de controle dos cânceres do colo do útero e de mama, e os dados mais atuais disponíveis.

O SIA/SUS é um sistema administrativo utilizado para realizar o pagamento pelo nível federal dos procedimentos ambulatoriais realizados no SUS. Dessa forma, prestadores de serviços ambulatoriais precisam seguir um fluxo de envio mensal da produçáo dos exames realizados para receberem o pagamento correspondente ${ }^{23}$. O Siscolo e o Sismama foram desenvolvidos para coletar as informaçôes epidemiológicas, provenientes de formulários padronizados de solicitação e laudo de exames de rastreamento e investigação diagnóstica, importantes para as açôes gerenciais de controle desses cânceres ${ }^{6}$. O Siscolo, Sismama e Siscan se relacionam com o SIA/SUS por gerarem o BPA-I, arquivo que contém informaçôes necessárias para o faturamento dos exames e que é importado pelo SIA/SUS ${ }^{6,8}$.

De acordo com a tabela de procedimentos do SUS ${ }^{22}$, os arquivos do BPA-I só podem ser aceitos pelo SIA/SUS se forem gerados exclusivamente pelo Siscolo, Sismama ou Siscan ${ }^{7,24,25}$. Além do arquivo de BPA-I, os sistemas de câncer também geram os dados epidemiológicos que devem ser encaminhados às coordenaçôes responsáveis pelo monitoramento das açôes de controle de câncer. Entretanto, diferenças no fluxo de envio dos dados desses sistemas $^{6}$ e do grau de implantaçáa $0^{26}$ acarretam diferenças no quantitativo de exames registrados (Figura 1).

Assim, os prestadores do SUS que utilizam o Siscolo e o Sismama, ao final do mês, geram dois arquivos, um com a produção ambulatorial contendo a relação dos exames 


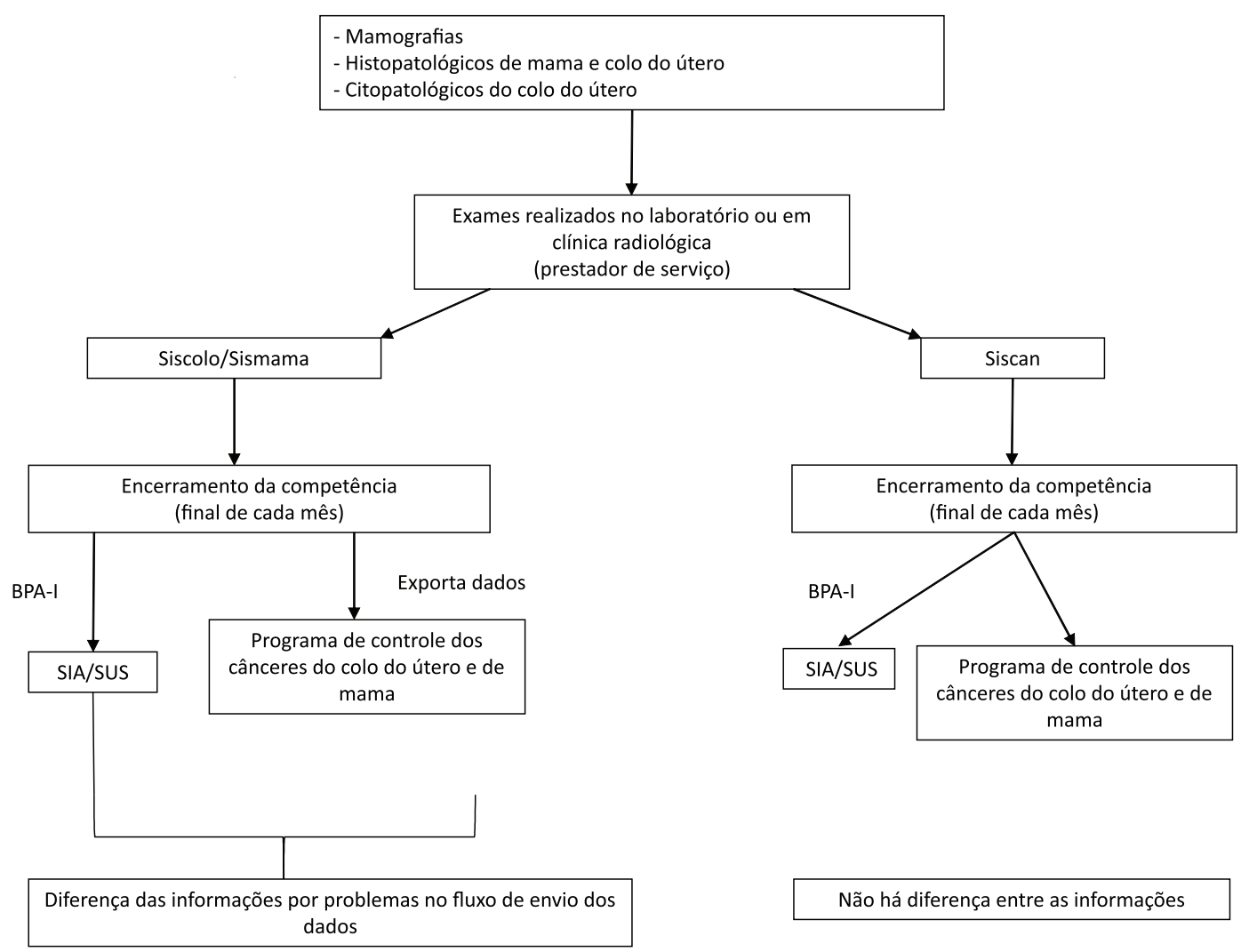

Figura 1. Fluxo das informações a partir da digitação no Siscolo, Sismama e Siscan e envio ao faturamento até a consolidação nas bases nacionais

Legendas: BPA-I = Boletim de produção ambulatorial; SIA/SUS = Sistema de Informaçôes Ambulatoriais do SUS; Siscolo = Sistema de Informação do Controle do Câncer do Colo do Útero; Sismama = Sistema de Informação do Controle do Câncer de Mama; Siscan = Sistema de Informaçáo de Câncer.

realizados, e outro com as informações epidemiológicas coletadas a partir dos formulários padronizados. Esses arquivos devem conter o mesmo quantitativo de exames, mas é possível haver pequenas diferenças, já que exames realizados em idade incompatível com as regras da tabela de procedimentos do SUS não são incluídos no arquivo de faturamento ${ }^{27}$; ou seja, são contabilizados no arquivo com informaçōes epidemiológicas, mas não no BPA-I. No entanto, problemas na rotina de envio dos arquivos podem ser a fonte de maior diferença entre os dados consolidados dos sistemas ${ }^{18}$.

Prestadores de serviços que utilizam o Siscan, ao final do mês, quando realizam o processo de encerramento da competência, geram o arquivo de BPA-I, que deve ser encaminhado ao setor de faturamento e, ao mesmo tempo, os dados epidemiológicos ficam disponíveis na base nacional, possibilitando avaliaçôes das açôes de controle dos cânceres do colo do útero e de mama, sem necessidade de envio dessas informaçôes ${ }^{8}$.

Os dados referentes ao Siscolo, ao Sismama e ao Siscan foram obtidos do Tabnet ${ }^{28}$, ferramenta de domínio público do Departamento de Informática do SUS (DATASUS), que disponibiliza, de forma não identificada, as informaçôes epidemiológicas desses sistemas.
O quantitativo de exames citopatológicos e histopatológicos do colo do útero de 2008 a 2014 foram obtidos do Tabnet do Siscolo, e os dos anos 2013 a 2019 do Tabnet do Siscan. Para o procedimento exame anatomopatológico - peça cirúrgica do colo uterino, a análise comparativa foi realizada a partir de 2014, apesar de o procedimento ser registrado no Siscan a partir de $2013^{7}$. Optou-se por inclui-lo em 2014, considerando que poucos serviços tinham iniciado o registro do procedimento de peça cirúrgica do colo do útero no Siscan em 2013 (Quadro 1).

O Siscolo incluía também exames de Monitoramento Externo da Qualidade (MEQ) dos exames citopatológicos, os quais eram cadastrados no SIA/SUS, entre 2008 e 2014, com o mesmo código do exame citopatológico. A partir de 2014, o procedimento de exames para o MEQ foi criado no SIA/SUS. Em virtude da dificuldade de identificar os exames de MEQ no SIA/SUS no período inicial, esse procedimento não foi incluído no estudo.

O quantitativo de mamografias e exames histopatológicos de mama foram obtidos do Tabnet do Sismama para o período de 2010 a 2014, e do Siscan para o período de 2013 a 2019 (Quadro 1).

Como os dados epidemiológicos do Siscolo e do Sismama estão disponíveis no Tabnet até 2014, os de 2013 
Quadro 1. Procedimentos, forma de registro nos sistemas de informação dos cânceres do colo do útero e de mama e período de obtenção dos dados por fonte

\begin{tabular}{|c|c|c|c|}
\hline \multicolumn{4}{|c|}{ Câncer de mama } \\
\hline SIA/SUS & Sismama/Siscan & \multicolumn{2}{|c|}{ Períodos } \\
\hline $\begin{array}{c}\text { Mamografia bilateral para rastreamento } \\
\text { (cód. 02.04.03.18-8) }\end{array}$ & $\begin{array}{l}\text { Mamografia de } \\
\text { rastreamento }\end{array}$ & \multirow{2}{*}{$\begin{array}{l}\text { Sismama } \\
(2010 \text { a 2014) }\end{array}$} & \multirow{2}{*}{$\begin{array}{c}\text { Siscan } \\
\text { (2013 a 2019) }\end{array}$} \\
\hline $\begin{array}{c}\text { Mamografia } \\
\text { (cód. 02.04.03.003-0) }\end{array}$ & $\begin{array}{l}\text { Mamografia } \\
\text { diagnóstica }\end{array}$ & & \\
\hline $\begin{array}{c}\text { Exame anatomopatológico de mama - } \\
\text { biópsia } \\
\text { (cód. 02.03.02.006-5) }\end{array}$ & $\begin{array}{l}\text { Exame histopatológico } \\
\text { biópsia }\end{array}$ & \multirow{2}{*}{$\begin{array}{l}\text { Sismama } \\
(2010 \text { a 2014) }\end{array}$} & \multirow{2}{*}{$\begin{array}{c}\text { Siscan } \\
\text { (2013 a 2019) }\end{array}$} \\
\hline $\begin{array}{c}\text { Exame anatomopatológico de mama - } \\
\text { peça cirúrgica } \\
\text { (cód. 02.03.02.007-3) }\end{array}$ & $\begin{array}{l}\text { Exame histopatológico } \\
\text { peça cirúrgica }\end{array}$ & & \\
\hline \multicolumn{4}{|c|}{ Câncer do colo do útero } \\
\hline SIA/SUS & Siscolo/Siscan & \multicolumn{2}{|c|}{ Períodos } \\
\hline $\begin{array}{l}\text { Exame citopatológico cervicovaginal/ } \\
\text { microflora (cód. 02.03.01.001-9) }\end{array}$ & Exame citopatológico & $\begin{array}{c}\text { Siscolo } \\
\text { (2008 a 2014) }\end{array}$ & $\begin{array}{c}\text { Siscan } \\
\text { (2013 a 2019) }\end{array}$ \\
\hline $\begin{array}{l}\text { Exame citopatológico cervicovaginal/ } \\
\text { microflora rastreamento } \\
\text { (cód. 02.03.01.008-6)* }\end{array}$ & $\begin{array}{l}\text { Exame citopatológico } \\
\text { de rastreamento** }\end{array}$ & $\begin{array}{l}\text { Não incluído no } \\
\text { Siscolo }\end{array}$ & $\begin{array}{c}\text { Siscan } \\
\text { (2013 a 2019) }\end{array}$ \\
\hline $\begin{array}{l}\text { Exame anatomopatológico do colo uterino } \\
\text { - biópsia (cód. 02.03.02.008-1) }\end{array}$ & $\begin{array}{c}\text { Exame histopatológico } \\
\text { - biópsia }\end{array}$ & $\begin{array}{c}\text { Siscolo } \\
\text { (2008 a 2014) }\end{array}$ & $\begin{array}{c}\text { Siscan } \\
\text { (2013 a 2019) }\end{array}$ \\
\hline $\begin{array}{c}\text { Exame anatomopatológico do colo uterino } \\
- \text { peça cirúrgica } \\
\text { (cód. 02.03.02.002-2) }\end{array}$ & $\begin{array}{l}\text { Exame histopatológico } \\
\text { - peça cirúrgica }\end{array}$ & $\begin{array}{c}\text { Não incluído no } \\
\text { Siscolo }\end{array}$ & $\begin{array}{c}\text { Siscan } \\
\text { (2013 a 2019) }\end{array}$ \\
\hline
\end{tabular}

Legendas: SIA/SUS = Sistema de Informaçôes Ambulatoriais do SUS; Siscolo = Sistema de Informação do Controle do Câncer do Colo do Útero; Sismama = Sistema de Informação do Controle do Câncer de Mama; Siscan = Sistema de Informação de Câncer.

(*) Procedimento criado no SIA/SUS a partir de setembro de 2014.

$\left.{ }^{(* *}\right)$ Até agosto de 2014, correspondeu ao procedimento 'exame citopatológico cervicovaginal/microflora (cód. 02.03.01.001-9)' no SIA/SUS.

a 2014 comuns ao Siscolo e Sismama ou ao Sismama e Siscan foram somados.

Os dados registrados no Siscolo e no Sismama a partir de 2015 não puderam ser incluídos na análise por não estarem disponíveis no Tabnet. Assim, nesses anos, compararam-se apenas os registros do Siscan ao SIA/SUS.

Os dados de produção dos exames do SIA/SUS foram obtidos dos arquivos de disseminaçáo, de acesso público, também disponíveis na página do DATASUS. Desse sistema, foram consideradas as variáveis: procedimento, competência do procedimento, código do estabelecimento no Cadastro Nacional de Estabelecimento de Saúde e código da UF do estabelecimento. O quantitativo dos procedimentos foi obtido pela frequência apresentada por minimizar possíveis problemas de subdimensionamento da produção ${ }^{29}$, exceto para mamografia, na qual se utilizou o quantitativo de exames registrados. Optou-se por não utilizar a frequência apresentada de mamografias, já que os exames com indicação clínica de diagnóstico sáo contabilizados por mama radiografada, podendo ser registrados até dois procedimentos ${ }^{22}$.
O quantitativo de exames registrados no SIA/SUS foi considerado como referência, por se tratar de um sistema de faturamento, no qual o risco de sub-registro é menor. Assim, os dados informados no SIA/SUS foram comparados aos informados no Siscolo, Sismama e Siscan por UF do prestador de serviço e ano da realização do exame.

A cobertura dos sistemas de controle de câncer foi calculada segundo a UF, tipo de exame e ano do registro, por meio da proporção de exames registrados nos sistemas de controle de câncer registrados também no SIA/SUS, conforme fórmula a seguir:

$$
\text { Cobertura }=\frac{\begin{array}{c}
\text { Exames no Sistema de Informação de } \\
\text { Controle do Câncer }
\end{array}}{\text { Exames no SIA/SUS }} * 100
$$

Foi calculado o indicador de perda de informação que indica a proporção de exames informados apenas no SIA/ SUS por tipo de exame, ano e UF, conforme a fórmula a seguir: 


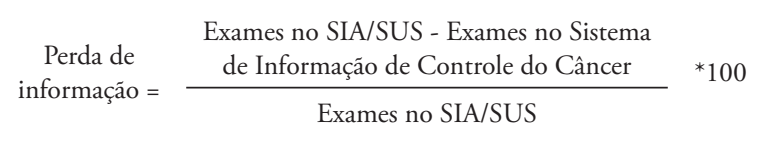

O indicador denominado "perda de informação" expressa a proporção de exames faltantes ou não nos dados dos sistemas de informaçôes epidemiológicas. Até 2014, refletia a proporção de exames faturados e que, por falhas no envio de dados, náo foram contabilizados no Tabnet do Siscolo e do Sismama. A partir de 2015, quando estáo disponíveis apenas os dados do Siscan, expressava a perda de informação ocorrida pela não utilizaçáo do Siscan e pela manutenção do uso do Siscolo e do Sismama, os quais não transmitem mais seus dados à base nacional.

Foi realizada análise comparativa entre os Estados e entre as Regiōes. Na apresentação gráfica por Estado, foram suprimidos os valores de perda da informação abaixo do percentil 2 e acima do percentil 98 para assegurar melhor visualização.

Em conformidade com as diretrizes da Resolução do Conselho Nacional de Saúde (CNS) no ${ }^{\circ}$ 510, de 7 de abril de $2016^{30}$, este estudo náo precisou ser submetido à avaliação do Comitê de Ética em Pesquisa (CEP) por utilizar exclusivamente dados secundários de acesso público e sem possibilidade de identificação individual.

\section{RESULTADOS}

Foram registrados no Siscolo e no Siscan, de 2008 a 2019, 97.353.388 exames citopatológicos e 692.157 exames histopatológicos. Já no Sismama e no Siscan, foram registrados, de 2010 a 2019, 28.300.349 mamografias e 270.790 histopatológicos.

No SIA/SUS, foram registrados, nesse mesmo período, 121.567.939 exames citopatológicos do colo do útero, 866.284 histopatológicos do colo do útero, 430.545 histopatológicos de mama e 37.938.255 mamografias.

No período analisado, verificou-se, para o Brasil, que o Siscolo apresentou as maiores coberturas com $80,1 \%$ para exames citopatológicos e $78,9 \%$ nos exames histopatológicos, mas com variaçóes importantes. A cobertura para os exames citopatológicos variou de $89,5 \%$ a 53,6\% nos anos de 2010 e 2015, respectivamente. Já em relação aos exames histopatológicos, a maior cobertura do sistema ocorreu nos anos de 2009 e 2010 (96,6\% em cada ano) e a menor cobertura, em 2015 (43,8\%).

Para o controle do câncer de mama, o sistema apresentou uma cobertura de $74,6 \%$ para mamografia e de $62,1 \%$ para exames histopatológicos no país. A melhor cobertura do sistema para a mamografia foi em 2012 (84,6\%) e para o histopatológico em 2011 (99,1\%); enquanto a pior cobertura foi em 2015 (46,6\%) para mamografia e em 2016 (35,8\%) para histopatológico.

Assim como observado para o país, o ano de 2015 foi o de maior diferença entre os registros nos sistemas para a maior parte das Regióes e exames. O exame citopatológico apresentou menor perda de informação dos sistemas epidemiológicos em relação ao de faturamento, em todas as Regiōes, até 2014 . As perdas foram superiores a 65\% nas Regiôes Sudeste e Norte em 2015. A Região Centro-Oeste apresentou padrão mais estável de 2008 a 2017; e, em 2018, houve mais registros no Siscan do que no SIA/SUS. A Região Sul atingiu perda em torno de $29 \%$ em 2014 com progressiva redução e alcançando maior registro no Siscan a partir de 2018 e a Região Nordeste atingiu em 2015 perda em torno de $38 \%$ com posterior redução e com inversão do cenário de perda em 2019 (Gráfico 1).

Quanto ao exame histopatológico do colo do útero, as Regiōes Centro-Oeste e Sudeste apresentaram maior percentual de dados no Siscolo entre 2008 e 2012, porém, a partir de então, demonstraram padróes diferenciados: no Centro-Oeste, houve perda de dados em relação ao SIA/SUS entre 2012 e 2017 e, em 2018, voltou a apresentar mais dados no Siscan; a Região Sudeste, em 2015, apresentou perdas de 76\% e manteve valores acima de $50 \%$ até o último ano de análise. As Regiōes Norte e Nordeste também apresentaram maiores perdas em 2015, respectivamente, $61 \%$ e $48 \%$, todavia apresentam declínio expressivo das perdas nos anos seguintes (Gráfico 1).

O exame histopatológico de mama foi o que apresentou mais variações. Até 2012, as Regiōes Centro-Oeste e Norte apresentaram mais informaçôes no Siscan, sendo que a Região Centro-Oeste manteve esse perfil durante todo o período analisado, excetuando os anos de 2015 e 2017, com 7\% de perda. Em 2012, a Região Sudeste inverteu o padrão, assim como a Região Norte, nos anos de 2013 e 2015, que teve perda superior a $60 \%$ neste último ano; porém, em 2018 e 2019, com registros no Siscan acima de 50\%. A Regiáo Sul, a partir de 2014, começava a apresentar um padrão de redução de perda. No Nordeste, houve uma tendência de perda crescente até 2016 com uma proporção de $52 \%$ e decrescente a partir desse ano, atingindo, em 2018, a proporção de $4 \%$ (Gráfico1).

A mamografia apresentava mais registros no Siscan para a Regiāo Centro-Oeste em todo no período. A Região Norte, em 2015, apresentava perda no Siscan superior a $75 \%$ quando comparada ao SIA/SUS ( $\mathrm{p}=0,948)$. A Região Sul apresentou um padrão de perda progressivo até 2013 e, a partir de então, iniciou a redução dessa proporção até 2019. No Nordeste, houve perda em todo o período, porém com redução a partir de 2016, atingindo, em 2019, 
um déficit de 3\%. No Sudeste, houve perda em todo período, sendo mais elevada em 2015 (70\%), Gráfico 1.

Avaliação das perdas de registros no Siscolo por Estado, tipo de exame e ano do exame mostram que o Espírito Santo foi o único que apresentou mais registros no programa de controle do câncer (Siscolo/Siscan) tanto para os exames citopatológicos quanto histopatológicos, de 2008 a 2019, com pequena perda apenas em 2015. Mato Grosso do Sul apresentou valores muito próximos entre os sistemas de informação, porém com um aumento localizado nos anos de 2013 e 2014. Alguns Estados apresentaram menores quantidades de registro no Siscan a partir de 2014, como Amazonas, Rondônia, Piauí, Rio de Janeiro, Rio Grande do Sul e São Paulo. Outros Estados que não tinham perda ou proporção menor de perda até 2013-2014 demonstraram aumento na perda de dados com reversão desse cenário em anos mais recentes: Distrito Federal, Minas Gerais, Pará, Paraná, Paraíba e Tocantins. Os Estados de Santa Catarina e Rio Grande do Norte apresentaram reduçáo da perda a partir de 2014 apenas para os exames citopatológicos do colo do útero (Gráfico 2).

$\mathrm{Na}$ avaliação dos Estados sobre as informaçôes referentes ao câncer de mama, exames mamográficos e histopatológicos foram os que apresentaram comportamentos bem diferenciados. Mato Grosso não mostrou perdas a partir de 2015 e Mato Grosso do Sul apresentou perdas para o histopatológico em relação ao SIA/SUS em 2010, 2012 e 2015. Tocantins, com exceção do ano de 2013, teve o mesmo padrão, com maior número de exames entre o Siscolo e o Siscan. Nos Estados do Maranhão, Goiás, Paraná, Santa Catarina, Sergipe e Paraíba, observa-se que ocorreu uma mudança no comportamento das perdas a partir dos anos 2013-2014, com diminuiçâo ou inversão da concentração de exames informados nos sistemas. São Paulo e Rio de Janeiro, a partir de 2015, apresentaram acentuada perda nos registros desses exames (Gráfico 3).

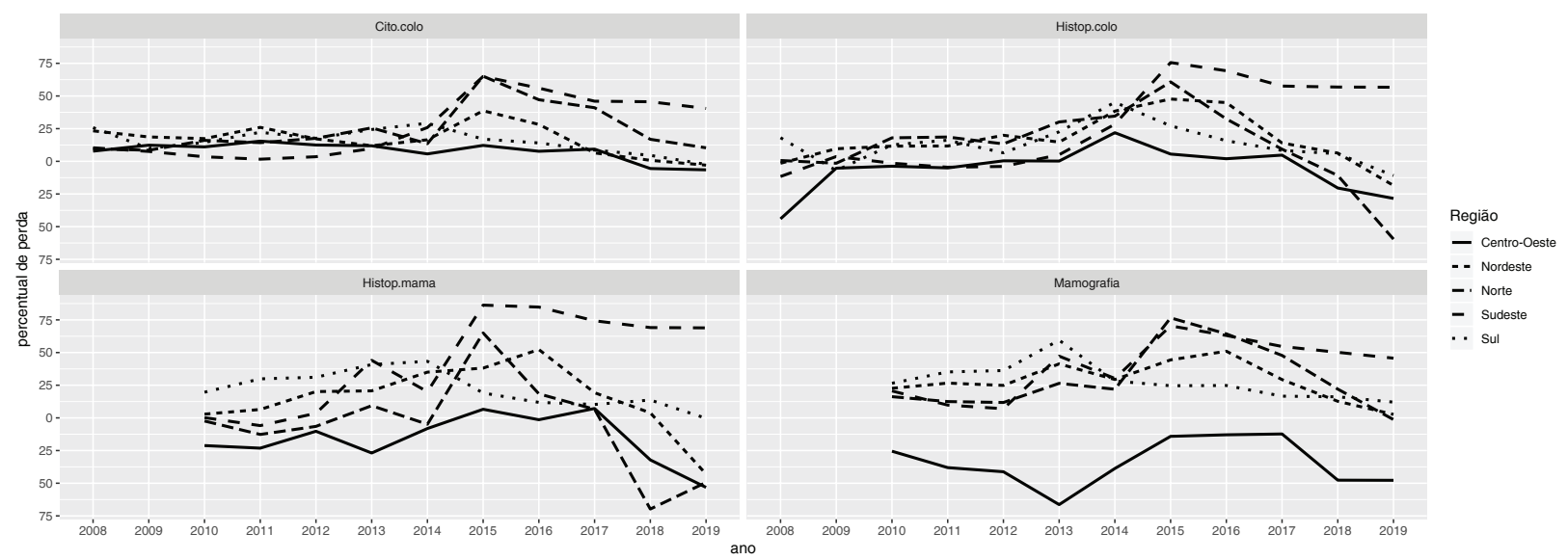

Gráfico 1. Perda de informação dos sistemas de informação dos cânceres do colo do útero e de mama segundo tipo de exame e região de atendimento. Brasil, 2008-2019

Fonte: Tabnet ${ }^{28}$.

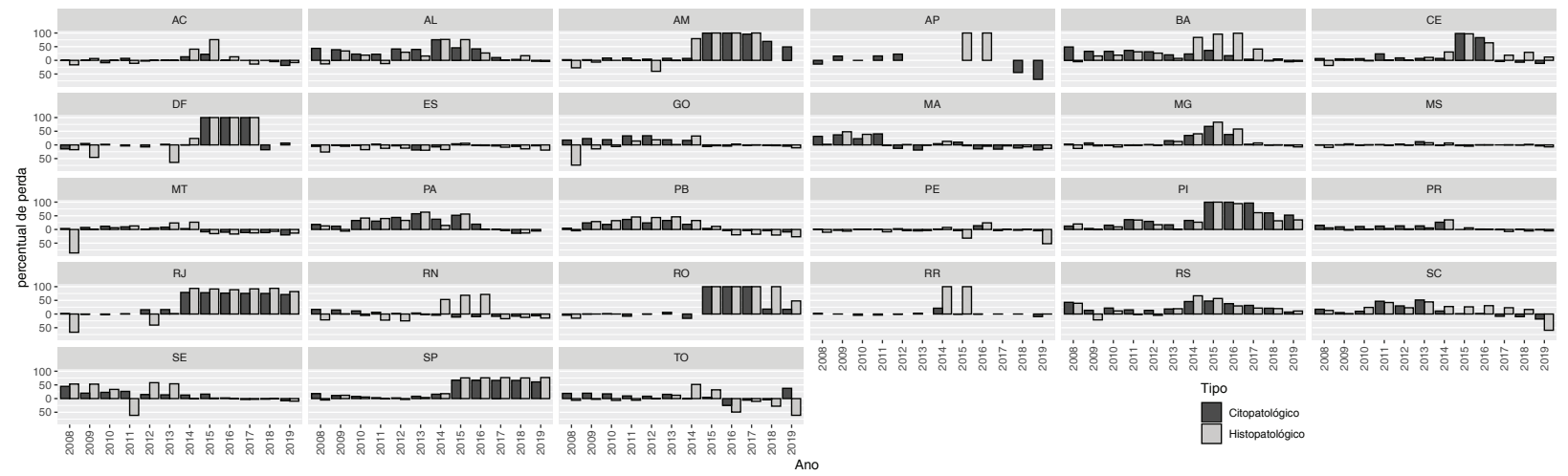

Gráfico 2. Perda de informação dos sistemas de informação do câncer do colo do útero segundo tipo de exame e Unidade da Federação de atendimento. Brasil, 2008-2019

Fonte: Tabnet ${ }^{28}$

Nota: Excluídos os exames citopatológicos do Amapá 2016 (-277,04\%) e 2017 (-662,95\%) e os exames histopatológicos do Rio de Janeiro dos anos de 2009 (-361,99\%), 2010 (-362,26\%) e 2011 (-168,55\%); Rondônia 2014 (-87,32\%); Distrito Federal 2010 (-105,07\%), 2011 (-267,95\%), 2012 (-100,40\%), 2018 $(-471,43 \%)$ e 2019 (-130,62\%); e Roraima 2011 (-100,0\%), Pará 2019 (-139,24\%). 


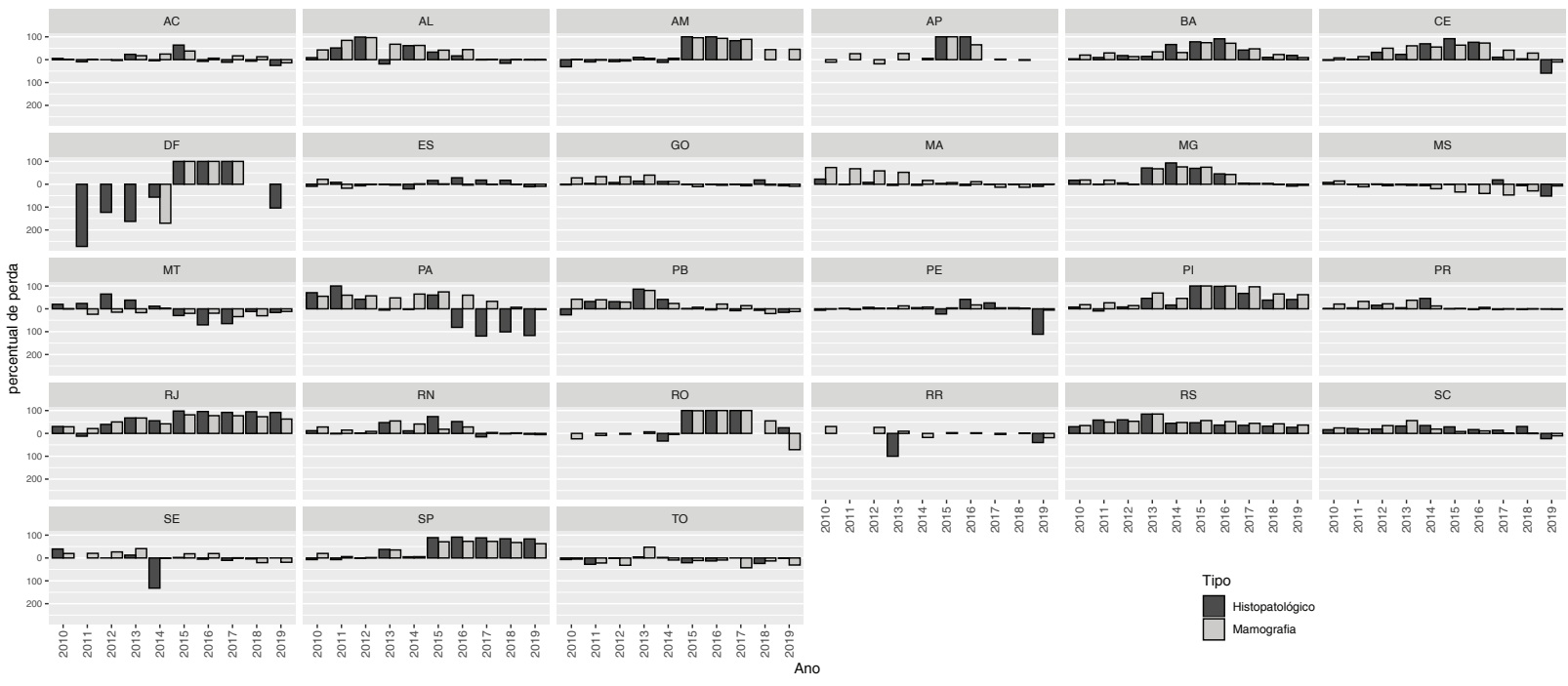

Gráfico 3. Perda de informação dos sistemas de informação do câncer de mama segundo tipo de exame e Unidade da Federação de atendimento. Brasil, 2008-2019

Fonte: Tabnet ${ }^{28}$.

Nota: Excluídas as mamografias de Roraima 2011 (-309,49\%), Amapá (-2229,26\%), Distrito Federal 2010 (-428,27\%), 2011 (-786,86), 2012 (-512,83\%), 2013 $(-470,32 \%), 2018(-1176,87 \%)$ e 2019 (-650,85\%). E exames histopatológicos de Sergipe $2011(-278,57)$, Distrito Federal 2010 (-291,76\%) e 2018 (-549,56\%).

\section{DISCUSSÃO}

A qualidade dos dados é um importante atributo em um sistema de informação e cuja inexatidão e discrepância ocasionam problemas no planejamento. Dessa forma, estudos que avaliem e identifiquem possíveis problemas nas informaçôes dos programas de saúde devem ser implementados ${ }^{31}$, propiciando aos gestores, sociedade organizada e demais usuários dessas informaçóes elementos para analisá-las criticamente ${ }^{21}$.

Entre as dimensôes de qualidade estudadas no Siscan, há referências na literatura para a completitude, confiabilidade, validade e oportunidade ${ }^{32,33}$, todavia não foi identificado estudo que avaliasse a dimensão cobertura. A análise da diferença dos registros de exames entre os sistemas de informaçôes epidemiológicas e de faturamento permitiu verificar a cobertura desses sistemas.

Os resultados deste estudo apontam que diferenças entre os registros nos sistemas de informaçôes epidemiológicas e de faturamento ocorrem desde o início da sua implantação e persistem até os dias atuais. As diferenças no número de registros variaram ao longo do período analisado, porém observou-se que a implantação do Siscan, que vem ocorrendo gradualmente desde o último trimestre de 2013, teve impacto significativo nas diferenças de registros. A partir de 2015, ano no qual os dados do Siscolo e Sismama deixaram de ser importados para a base nacional, a maioria dos estados registrou maiores diferenças nas informaçôes, ainda que, para alguns, se verifique ganho das informaçôes epidemiológicas com a implantação do Siscan.
O exame citopatológico do colo do útero, procedimento com maior volume de produção entre os analisados, foi o que apresentou maior cobertura de informação no período, possivelmente pelo Siscolo ter sido o primeiro implantado no país e seu uso estar mais consolidado na rotina nos serviços de citopatologia. Entre os anos de 2008 e 2014, essa diferença pode ser ainda menor, uma vez que no SIA/SUS estão contabilizados em um mesmo procedimento os exames de monitoramento externo que não são incluídos na tabulação dos dados do Siscolo no Tabnet. Entretanto, a implantação do MEQ era limitada a poucos Estados ${ }^{34}$.

Os exames anatomopatológicos apresentaram elevadas proporções de perda de informação, e consequentemente pior cobertura, o que compromete a avaliação dos diagnósticos realizados. Isso ocorre possivelmente pelo registro desses exames em outro procedimento no SIA/ SUS, utilizando o código de exame anatomopatológico realizado em outros sítios anatômicos (020302003-0 exame anatomopatológico para congelamento/parafina por peça cirúrgica ou biópsia, exceto colo uterino e mama). Embora a tabela de procedimentos do SUS explicite que esse código não deva ser utilizado para faturamento de anatomopatológico do colo do útero (C53) e de mama (C50), a crítica ${ }^{22}$ ao diagnóstico informado; ou seja, a $10^{a}$ Classificação Internacional de Doenças e Problemas Relacionados à Saúde $\left(\right.$ CID-10) ${ }^{35}$, não era aplicada e observava-se que alguns laboratórios registravam as CID C50 e C53 no procedimento "exame anatomopatológico para congelamento/parafina por peça cirúrgica ou biópsia, exceto colo uterino e mama”. 
Como desde 2015 não há mais informaçóes do Siscolo e Sismama disponíveis para serem comparadas à produção apresentada no SIA/SUS, ao se observarem mais registros de informaçôes no Siscan, pode-se inferir que, nessas localidades, este esteja com maior grau de implantação/ cobertura. Por outro lado, altas proporçôes de perda dos dados epidemiológicos podem estar associadas ao maior uso do Siscolo e Sismama e a proporção de perda refletiria provavelmente a ausência na disponibilidade dessas bases e não uma perda real.

Nos anos de 2018 e 2019, observou-se queda na perda de informaçáo na maior parte dos Estados, provável reflexo da consolidação da implantação do Siscan. No entanto, Rio de Janeiro e Sáo Paulo ainda apresentam elevados percentuais de perda em todos os anos, o que deve ser explicado pela manutenção do uso do Siscolo e Sismama pelos laboratórios de grande porte, que possuem sistemas de informação próprios e que não exportam suas informações ao Siscan ${ }^{26}$.

Em alguns Estados, foi possível observar valores de perda negativos; ou seja, mais registros nos sistemas epidemiológicos do que nos de faturamento. Possíveis explicaçôes são o pagamento direto aos serviços por Estados e municípios, sem a utilização dos recursos federais $^{29,36}$, onde os gestores locais condicionam o pagamento dos serviços ao registro das informaçóes no sistema epidemiológico, à glosa de procedimentos e à perda do prazo de apresentaçáo dos procedimentos realizados ${ }^{37} \mathrm{e}$, portanto, sem registro no SIA/SUS e registros realizados por instituições com orçamento próprio, como hospitais federais e universidades. A diferença proveniente de glosas e perda de prazo implicam em um sub-registro no SIA/ SUS, enquanto as diferenças por pagamento direto ou por informaçôes de instituições com orçamento próprio proporcionam ganho de registros nos sistemas de controle de câncer e podem proporcionar uma compensação nas perdas reais.

Os dados do Siscolo e do Sismama têm sido utilizados no Brasil no monitoramento das açóes de detecção precoce desses cânceres e para subsidiar açóes e políticas públicas ao longo dos anos. Neste estudo, pode ser observado que o tempo para a consolidaçáo da sua utilizaçáo foi longo, uma vez que as menores perdas foram apresentadas pelo Siscolo, iniciado em 1999, e que o demorado processo de implantaçáo do Siscan impactou de forma importante na perda de dados no Brasil, comprometendo as avaliações das açóes de detecção precoce no país e a utilização dos dados para produção de conhecimento por meio de pesquisa científica. A descontinuidade da alimentação da base nacional antes da interrupção do uso do Siscolo e do Sismama e a indisponibilidade de uma ferramenta de integração entre os sistemas de laboratórios de grande porte e o Siscan podem ser apontados como principais fatores para as grandes diferenças encontradas.

É recomendável que análises utilizando dados desses sistemas sejam antecedidas por uma avaliaçáo crítica da cobertura do sistema em cada local e período analisado e como podem impactar no tipo de análise realizada. Estudos que tenham por objetivo estimar quantitativos de procedimentos ou custos devem preferencialmente utilizar dados do SIA/SUS. A avaliação da série histórica por UF deve ser acompanhada particularmente pelos gestores que devem priorizar, junto à sua equipe, estratégias para melhorar a cobertura dos sistemas de controle do câncer e consequentemente a qualidade das análises realizadas a partir delas.

O estudo tem como limitação a indisponibilidade de informaçáo dos dados do Siscolo e do Sismama dos Estados que ainda os utilizam. Essa ausência de informação poderá impactar na análise de alguns Estados.

O Brasil está avançando na implantação integral do Siscan em todo território nacional e, com isso, gradualmente as diferenças de registros entre os sistemas estão sendo superadas, já com menores patamares em 2019. Assim, espera-se que, nos próximos anos, os dados do Siscan possam refletir satisfatoriamente a cobertura das açốes de detecção precoce dos cânceres do colo do útero e de mama no SUS, subsidiando adequadamente a gestão e a pesquisa.

\section{CONCLUSÃO}

A perda de informação dos sistemas que registram procedimentos de rastreamento e diagnóstico dos cânceres do colo do útero e de mama ocorre desde a implantação desses sistemas, com impacto na utilização dos dados para análise, monitoramento e planejamento das açôes de controle do câncer. O período inicial de implantação de um novo sistema mostrou-se crítico. O Siscan, por ser um sistema on-line, minimizaria as perdas de informação, porém observou-se o efeito contrário nos primeiros anos de sua utilização, com grandes perdas de informação. No entanto, a implantação gradual do sistema nos últimos anos tem se mostrado potencialmente vantajosa na redução da perda de registros.

\section{CONTRIBUIÇÕES}

Jeane Tomazelli, Caroline Madalena Ribeiro e Maria Beatriz Kneipp Dias contribuíram substancialmente na concepção e/ou planejamento do estudo; na obtenção, análise e/ou interpretação dos dados; na redação e revisão crítica; e aprovaram a versão final a ser publicada. 


\section{DECLARAÇÃO DE CONFLITO DE INTERESSES}

Nada a declarar.

\section{FONTES DE FINANCIAMENTO}

Não há.

\section{REFERÊNCIAS}

1. Instituto Nacional de Câncer José Alencar Gomes da Silva. Estimativa 2020: incidência de câncer no Brasil [Internet]. Rio de Janeiro: INCA; 2020 [acesso $2020 \mathrm{abr}$ 15]. Disponível em: https://www.inca.gov.br/sites/ufu. sti.inca.local/files//media/document//estimativa-2020incidencia-de-cancer-no-brasil.pdf

2. Miller AB, Nazeer S, Fonn S, et al. Report on consensus conference on cervical cancer screening and management. Int J Cancer. 2000;86(3):440-7. doi: https://doi. org/10.1002/(sici)1097-0215(20000501)86:3<440::aidijc22>3.0.co;2-a 3

3. International Agency for Research on Cancer. Breast cancer screening [Internet]. Lyon, FR: IARC; 2016. [cited 2020 Apr 15]. (IARC Handbooks of Cancer Prevention; vol. 15). Available from: https://publications. iarc.fr/Book-And-Report-Series/Iarc-Handbooks-OfCancer-Prevention/Breast-Cancer-Screening-2016

4. Australian Institute of Health and Welfare. BreastScreen Australia monitoring report 2019 [Internet]. Canberra, AU: AIHW; c2019 [cited 2020 Mar 10]. (Cancer series; no. 127, Cat. no. CAN 128). Available from: https:// apo.org.au/sites/default/files/resource-files/2019-10/ apo-nid262271.pdf

5. Canadian Partnership Against Cancer. The 2012 cancer system performance report [Internet]. Toronto, CA: Canadian Partnership Against Cancer; 2012 Dec [cited 2020 Mar 10]. Available from: https://s22457.pcdn. co/wp-content/uploads/2019/12/2012-cancer-systemperformance-report-EN.pdf

6. Instituto Nacional de Câncer. Sistemas de informação do controle do câncer de mama e do colo do útero (SISMAMA) e do câncer do colo do útero (SISCOLO): manual gerencial. Rio de Janeiro: INCA; 2011.

7. Ministério da Saúde (BR). Portaria no 3.394, de 30 de dezembro de 2013. Institui o Sistema de Informaçáo de Câncer (SISCAN) no âmbito do Sistema Único de Saúde (SUS) [Internet]. Diário Oficial da Uniāo. 2013 dez 31 [acesso 2020 dez 8]; Seção I:57. Disponível em: http://bvsms.saude.gov.br/bvs/saudelegis/gm/2013/ prt3394_30_12_2013.html

8. Instituto Nacional de Câncer José de Alencar Gomes da Silva. Sistema de informação do câncer. manual preliminar para apoio à implantação [Internet]. Rio de Janeiro: INCA; 2013 [acesso $2020 \mathrm{dez}$ 8]. Disponível em: https://www.inca.gov.br/sites/ufu.sti.inca.local/ files//media/document//sistema-informacao-cancermanual.pdf

9. Tomazelli J, Azevedo e Silva G. Rastreamento do câncer de mama no Brasil: uma avaliação da oferta e utilização da rede assistencial do Sistema Único de Saúde no período 2010-2012. Epidemiol Serv Saúde. 2017;26(4):713-24. doi: https://doi.org/10.5123/ S1679-49742017000400004

10. Dias MBK, Tomazelli J, Assis M. Rastreamento do câncer de colo do útero no Brasil: análise de dados do Siscolo no período de 2002 a 2006. Epidemiol Serv Saúde. 2010;19(3):293-306. doi: http://doi.org/10.5123/ S1679-49742010000300011

11. Corrêa CSL, Lima AS, Leite ICG, et al. Rastreamento do câncer do colo do útero em Minas Gerais: avaliaçáo a partir de dados do Sistema de Informaçáo do Câncer do Colo do Útero (SISCOLO). Cad Saúde Colet. 2017;25(3):315-23. doi: https://doi.org/10.1590/1414462X201700030201

12. Costa RFA, Longatto-Filho A, Vazquez FL, et al. Trend analysis of the quality indicators for the Brazilian cervical cancer screening programme by region and state from 2006 to 2013. BMC Cancer. 2018;18:126. doi: https:// doi.org/10.1186/s12885-018-4047-9

13. Costa RFA, Longatto-Filho A, Pinheiro C, et al. Historical analysis of the Brazilian cervical cancer screening program from 2006 to 2013: a time for reflection. PLoS ONE. 2015;10(9):e0138945. doi: https://doi.org/10.1371/ journal.pone. 0138945

14. Ribeiro CM, Azevedo e Silva G. Avaliação da produção de procedimentos da linha de cuidado do câncer do colo do útero no Sistema Único de Saúde do Brasil em 2015. Epidemiol Serv Saude. 2018;27(1):e20172124. doi: https://doi.org/10.5123/S1679-49742018000100004

15. Silva DSM, Silva AMN, Brito LMO, et al. Rastreamento do câncer do colo do útero no Estado do Maranháo, Brasil. Ciênc Saúde Coletiva. 2014;19(4):1163-70. doi: https://doi.org/10.1590/1413-81232014194.00372013

16. Azevedo e Silva G, Bustamante-Teixeira MT, Aquino EML, et al. Acesso à detecção precoce do câncer de mama no Sistema Único de Saúde: uma análise a partir dos dados do Sistema de Informaçôes em Saúde. Cad Saúde Pública. 2014;30(7):1537-50. doi: https://doi. org/10.1590/0102-311X00156513

17. Bortolon PC, Silva MAF, Côrrea FM, et al. Avaliação da qualidade dos laboratórios de citopatologia do colo do útero no Brasil. Rev Bras Cancerol. 2012;58(3):435-44. doi: https://rbc.inca.gov.br/revista/index.php/revista/ article/view/600/369

18. Instituto Nacional de Câncer José de Alencar Gomes da Silva. Monitoramento das açôes de controle dos cânceres do colo do útero e da mama. Informativo Detecção Precoce [Internet]. $2010 \mathrm{abr} / \mathrm{jun}$ [acesso $2020 \mathrm{dez}$ 
8];(2):1-10. Disponível em: http://bvsms.saude.gov.br/ bvs/publicacoes/inca/informativo_detec_precoce_2.pdf

19. Tomazelli J, Migowski A, Ribeiro CM, et al. Assessment of actions for breast cancer early detection in Brazil using process indicators: a descriptive study with Sismama data, 2010-2011. Epidemiol Serv Saúde. 2017;26(1). doi: https://doi.org/10.5123/S167949742017000100007

20. Corrêa CSL, Pereira LC, Leite ICG, et al. Rastreamento do câncer de mama em Minas Gerais: avaliação a partir de dados dos sistemas de informaçôes do Sistema Único de Saúde. Epidemiol Serv Saude. 2017;26(3):481-49. doi: https://doi.org/10.5123/S1679-49742017000300006

21. Lima CRA, Schramm JMA, Coeli CM, et al. Revisão das dimensôes de qualidade dos dados e métodos aplicados na avaliação dos sistemas de informação em saúde. Cad Saúde Pública. 2009;25(10):2095-109. doi: https://doi. org/10.1590/S0102-311X2009001000002

22. Ministério da Saúde (BR). Portaria no 2.848, de 06 de novembro de 2007. Publica a Tabela de Procedimentos, Medicamentos, Órteses, Próteses e Materiais Especiais - OPM do Sistema Único de Saúde. Diário Oficial da União. 2007 nov 7; Seção 1:54.

23. Ministério da Saúde (BR), Secretaria de Atenção à Saúde, Departamento de Regulação, Avaliação e Controle, Coordenação Geral de Sistemas de Informação. Manual técnico operacional SIA/SUS: sistema de informaçôes ambulatoriais: aplicativos auxiliares e de captação da produção ambulatorial APAC Magnético -BPA Magnético -VERSIA - DE-PARA -FPO Magnético: orientaçôes técnicas [Internet]. Brasília, DF: Ministério da Saúde; 2010 [acesso 2020 dez 8]. Disponível em: http:// www1.saude.rs.gov.br/dados/1273242960988Manual_ Operacional_SIA2010.pdf

24. Ministério da Saúde (BR), Secretaria de Assistência à Saúde. Portaria no 408, de 30 de agosto de 1999. Determinar que o pagamento dos procedimentos referentes à citopatologia, histopatologia, e controle de qualidade ficará vinculado à prestaçáo de informaçóes necessárias ao monitoramento e avaliação das atividades de controle do câncer de colo de útero no Brasil, por intermédio de BPA em meio magnético, gerado exclusivamente pelo Sistema definido nesta Portaria. Diário Oficial da Uniāo. 1999 ago 2; Seção 1:14.

25. Ministério da Saúde (BR), Secretaria de Assistência à Saúde. Portaria no 779, de 31 de dezembro de 2008. Define como sistema de informação oficial do Ministério da Saúde, a ser utilizado para o fornecimento dos dados informatizados dos procedimentos relacionados ao rastreamento e a confirmação diagnóstica do câncer de mama, o Sistema de Informação do Controle do Câncer de Mama (SISMAMA) [Internet]. Diário Oficial da Uniáo. 2009 jan 2 [acesso 2020 dez 8]; Seção 1:38. Disponível em: http://bvsms.saude.gov.br/bvs/ saudelegis/sas/2008/prt0779_31_12_2008.html
26. Instituto Nacional de Câncer José de Alencar Gomes da Silva. Monitoramento das açóes de controle dos cânceres do colo do útero e da mama. Informativo Detecção Precoce [Internet]. 2019 jul/dez [acesso 2020 dez 8];10(2):1-7. Disponível em: https://www.inca. gov.br/sites/ufu.sti.inca.local/files//media/document// informativo-deteccao-precoce-numero2-2019.pdf

27. SIGTAP: Sistema de Gerenciamento de Tabela de Procedimentos, Medicamentos e OPM do SUS [Internet]. Brasília, DF: DATASUS. [2007] - [acesso 2020 dez 28]. Disponível em: http://sigtap.datasus.gov. br/tabela-unificada/app/sec/inicio.jsp

28. TABNET [Internet]. Brasília, DF: DATASUS. c2008 [acesso 2020 maio 28]. Disponível em: https://datasus. saude.gov.br/informacoes-de-saude-tabnet/

29. Pires M, Vieira R. Medidas preliminares de produção na saúde pública. Carta Conjunt [Internet]. 2017 [acesso 2020 maio 4];(37). Disponível em: http://www.ipea. gov.br/portal/images/stories/PDFs/conjuntura/171027_ cc_37_nt_1.pdf

30. Conselho Nacional de Saúde (BR). Resolução no 510, de 7 de abril de 2016. Dispóe sobre as normas aplicáveis a pesquisas em Ciências Humanas e Sociais cujos procedimentos metodológicos envolvam a utilização de dados diretamente obtidos com os participantes ou de informaçóes identificáveis ou que possam acarretar riscos maiores do que os existentes na vida cotidiana, na forma definida nesta Resolução [Internet]. Diário Oficial da União. 2016 maio 24 [acesso 2020 dez 8]; Edição98, Seção I:44. Disponível em: https://www. in.gov.br/materia/-/asset_publisher/Kujrw0TZC2Mb/ content/id/22917581

31. Silveira MH. Consideraçóes sobre o sistema de informação no setor saúde. Rev Saúde Pública. 1974;8(1):119-28. doi: https://doi.org/10.1590/S003489101974000100013

32. Girianelli VR, Thuler LCS, Silva GA. Qualidade do Sistema de Informaçáo do Câncer do Colo do Útero no estado do Rio de Janeiro. Rev Saúde Pública. 2009;43(4):580-8. doi: https://doi.org/10.1590/S003489102009005000043

33. Sartori MCS. Avaliação da qualidade do sistema de informaçáo do câncer do colo do útero (SISCOLO/ SISCAN) [dissertação na Internet]. São Paulo: Universidade Estadual Paulista; 2016 [acesso 2020 dez 7]. Disponível em: https://repositorio.unesp.br/ bitstream/handle/11449/139562/sartori_mcs_me_bot. pdf?sequence $=3$

34. Conselho Nacional de Secretários de Saúde (BR). Nota técnica 22: proposta de consolidaçẫo do Cartão Nacional de Saúde - "Cartão SUS": atualizaçâo das notas técnicas 29/2010 e 32/2010 de 06 de agosto e 13 de setembro de 2010 [Internet]. Brasília, DF: CONASS; 2011.

35. Organização Mundial da Saúde (OMS). Classificação Estatística Internacional de Doenças e Problemas 
Relacionados à Saúde. 10. ed. São Paulo: Editora da USP; 2017.

36. Silva CR, Carvalho BG, Cordoni Júnior L, et al. Dificuldade de acesso a serviços de média complexidade em municípios de pequeno porte: um estudo de caso. Ciênc Saúde Colet. 2017;22(4):1109-20. doi: https:// doi.org/10.1590/1413-81232017224.27002016

37. Santos CR. Projeto de informatização das solicitações de revisão e cobrança administrativa da produção ambulatorial e hospitalar do SUS/RS [trabalho de conclusão de curso]. Porto Alegre, RS: Fundação Oswaldo Cruz; Grupo Hospitalar Conceição; 2012.

Recebido em 15/2/2021

Aprovado em 8/6/2021 